\title{
Broadband Laser-Based Infrared Detector for Gas Chromatography
}

\section{Tomberg, Teemu}

2020-11-03

Tomberg , T , Vuorio , N , Hieta , T , Jussila , M , Hartonen, K, Vainio , M , Mikkonen , T , Toivonen, J , Riekkola , M-L , Halonen , L \& Metsälä , M 2020 , ' Broadband Laser-Based Infrared Detector for Gas Chromatography ' , Analytical Chemistry , vol. 92 , no. 21 , pp. 14582-14588 . https://doi.org/10.1021/acs.analchem.0c02887

http://hdl.handle.net/10138/324225

https://doi.org/10.1021/acs.analchem.0c02887

cc_by

publishedVersion

Downloaded from Helda, University of Helsinki institutional repository.

This is an electronic reprint of the original article.

This reprint may differ from the original in pagination and typographic detail.

Please cite the original version. 


\section{Broadband Laser-Based Infrared Detector for Gas Chromatography}

Teemu Tomberg, Niko Vuorio, Tuomas Hieta, Matti Jussila, Kari Hartonen, Markku Vainio, Tommi Mikkonen, Juha Toivonen, Marja-Liisa Riekkola, Lauri Halonen, and Markus Metsälä*

Cite This: Anal. Chem. 2020, 92, 14582-14588

Read Online

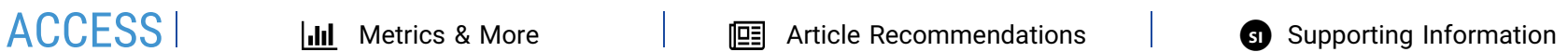

ABSTRACT: Cantilever-enhanced photoacoustic spectroscopy coupled with gas chromatography is used to quantitatively analyze a mixture of alcohols in a quasi-online manner. A full identification and quantification of all analytes are achieved based on their spectral fingerprints using a widely tunable continuous-wave laser as a light source. This can be done even in the case of interfering column/septum bleed or simultaneously eluted peaks. The combination of photoacoustic spectroscopy and gas chromatography offers a viable solution for compact and portable instruments in applications that require straightforward analyses with no consumables.

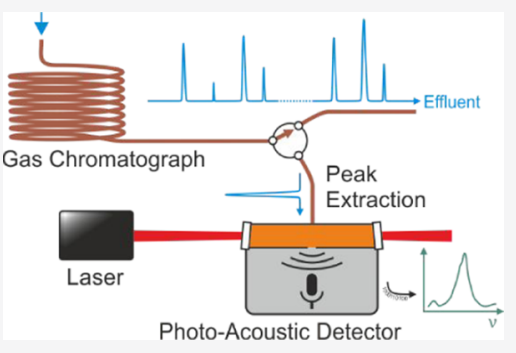

$\mathrm{L}$ aser spectroscopy based trace gas detectors are known for their high sensitivity and selectivity. Most of these instruments can be used to detect only small molecules, such as $\mathrm{CH}_{4}$ or $\mathrm{CO}_{2}$, which possess well-resolved and characteristic spectral features. Large molecules, such as $\mathrm{C}_{8} \mathrm{H}_{18} \mathrm{O}$, do not have identifiable spectral patterns inside the narrow spectral range of a typical semiconductor laser. If there is a complex mixture of large molecules, then the spectra become too complicated even for the current broadband or widely tunable laser technologies due to significant overlap and resemblance of different spectral features.

Gas chromatography (GC) offers the possibility to separate the compounds before the detection. Capillary GC, commonly coupled with detectors, such as flame ionization detectors (FIDs) or mass spectrometers (MSs), is a mature and highly reliable method for laboratory analyses of volatile organic compounds (VOCs). Many official standardized methods rely on GC combined with the mass spectrometry (MS) (gas chromatography-mass spectrometry (GC-MS)), as it currently provides the most universal platform for the detection and quantitative analysis of chemical compounds. Standard GC-MS instruments are laboratory-based and not directly suitable for mobile operations. However, smaller GC-MS analyzers are also available and these are used in field applications. The trade-off in portable GC-MS is normally lower sensitivity and inferior mass separation compared to a laboratory instrument.

The application of modern laser absorption spectroscopy (LAS) as a GC detector could offer new possibilities to analytical chemistry, especially considering mobile instruments. LAS with the recently developed broadband or broadly tunable light sources provides similar identification and selectivity as Fourier transform infrared (FTIR) spectrometers with a significantly improved detection sensitivity. Although FTIR spectrometers possess unrivaled molecular identification capabilities, their detection sensitivity is orders of magnitude worse than that of MSs or even FIDs. This has restricted their adoption as GC detectors. The reason for low sensitivity arises from the mismatch of sample volumes in GCs and FTIRs. The sample gas volume in GCs is typically in the microliter range, approximated as a volume equal to the full width at half-height (FWHH) of peaks emerging from a common capillary column. It is impossible to have a sufficient absorption path length with incoherent light sources in these small gas volumes. ${ }^{1,2}$ The detection sensitivity of an optical absorption-based detector depends linearly on the optical path length according to the Beer-Lambert law. Typical FTIR detectors, which have been developed for coupling to GC, employ lightpipe absorption cells that are about $10 \mathrm{~cm}$ long and $100 \mu \mathrm{L}$ in volume, which is insufficient for a good detection sensitivity. ${ }^{1,2}$ Vacuum ultraviolet (VUV) detectors have recently improved the detection performance of lightpipes by utilizing the electronic transitions in the VUV that are much stronger than the vibrational ones in the infrared region. This offers equally improved detection sensitivity in a small sample volume., The VUV detectors are currently one of the best alternatives to MS detection in qualitative chemical analysis. However, a sensitive mid-infrared detector would allow access to a wealth of additional chemical information in the analysis of the eluted peaks. Infrared LAS detectors can offer optical path lengths from tens to thousands of meters in small enough volumes due to the high coherence and brightness of lasers. On the other hand, some LAS techniques, such as photoacoustic spectroscopy (PAS), deliver orders of magnitude better detection

Received: July 7, 2020

Accepted: October 7, 2020

Published: October 21, 2020 


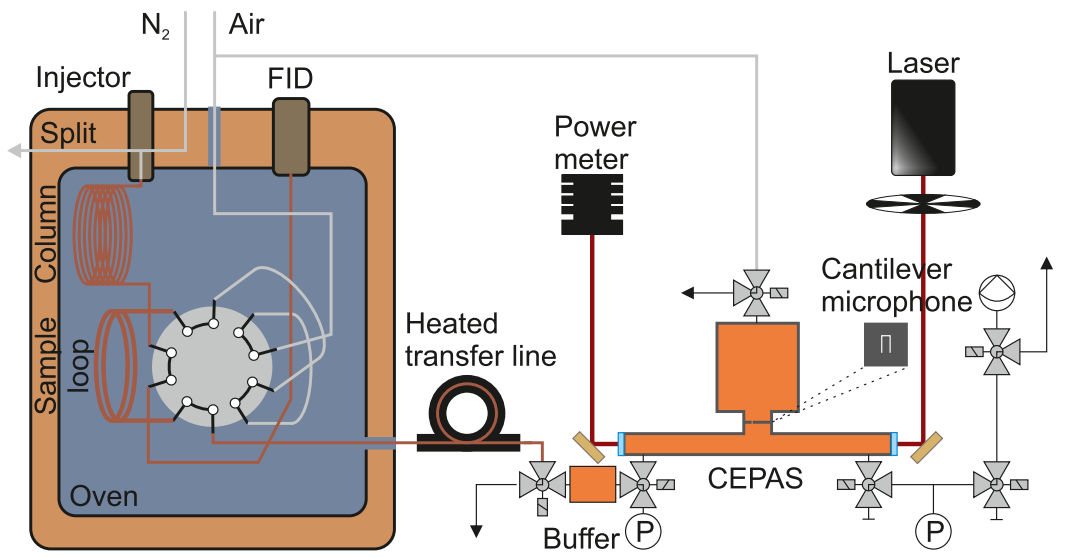

Figure 1. Schematic picture of the GC-PAS system. Part of the column effluent is sampled to the CEPAS detector by a sample loop and a rotary valve. P: pressure sensor.

sensitivity compared to FTIR detectors even with a short absorption path length.

Despite the advantages that the combination of GC and LAS can potentially provide, this approach has so far gained little attention. One of the main reasons has been the narrow spectral coverage of traditional laser sources, resulting in the incapability to simultaneously identify a wide breadth of compounds. Whereas an FTIR spectrometer employs a broadband incoherent light source with a typical optical detection band from 6500 to $600 \mathrm{~cm}^{-1}$, a typical tuning range of a single-mode laser is a few $\mathrm{cm}^{-1}$. As a result, most LASbased trace gas analyzers have been limited to measuring simultaneously one to three different low-molecular-weight compounds. However, in recent years, new broadband and tunable laser sources have emerged, including mid-infrared frequency combs ${ }^{5}$ and external-cavity quantum cascade lasers (EC-QCLs). ${ }^{6}$ As an example, mid-infrared frequency combs can provide simultaneous coherent multimode operation over $1300 \mathrm{~cm}^{-1}$ range centered around $2500 \mathrm{~cm}^{-1}$, whereas ECQCLs can be tuned over $400 \mathrm{~cm}^{-1}$ in the $1100 \mathrm{~cm}^{-1}$ region. ${ }^{8}$ This development, together with a suitable separation technique, such as gas chromatography, enables for the first time to develop a truly multicomponent LAS-based trace gas analyzer, also for larger-molecular-weight compounds.

To the best of our knowledge, two types of modern LASbased infrared detectors for GC have been published. One was developed by $\mathrm{Wu}$ et al., ${ }^{9}$ who used a quantum cascade laser (QCL) coupled to a hollow optical waveguide. The optical waveguide is similar to a GC capillary and increases the absorption path length tenfold compared to lightpipes, while maintaining sample volumes in the microliter range. In a more recent work, Mengali et al. used the same principle but employed a widely tunable EC-QCL to allow better spectral identification. ${ }^{10}$ With the absorption path length in the order of meters, the reported hollow waveguide spectrometers leave room for improvement in terms of detection sensitivity compared to the state-of-the-art LAS detectors. In another type of work, Zare et al. ${ }^{11}$ coupled a standard cavity ring-down spectrometer (CRDS) to a gas chromatograph through a combustor, resulting in the analysis of ${ }^{13} \mathrm{C} /{ }^{12} \mathrm{C}$ isotope ratios of organic compounds. Their typical CRDS had several orders of magnitude too large sample volume to provide required sensitivity for trace gas analysis.

Photoacoustic spectroscopy is an attractive technique for GC detectors. The combination was investigated at the turn of 1980s with gas lasers and packed columns. ${ }^{12,13}$ The research did not spark further interest mostly due to technical limitations of laser technology and the proliferation of capillary columns, which decreased the eluted gas volumes dramatically. Modern photoacoustic spectroscopy, on the other hand, can offer high detection capabilities ${ }^{14}$ in a small sample volume, as PAS does not depend on the absorption path length in the same way as transmission spectroscopy. ${ }^{15}$ For example, in quartz-enhanced photoacoustic spectroscopy (QEPAS), the effective absorption path length is less than a millimeter, ${ }^{16}$ yet QEPAS detectors have achieved a sub-ppb level detection limit in the volume mixing ratio. ${ }^{17}$ Another photoacoustic technique, cantilever-enhanced photoacoustic spectroscopy (CEPAS), has been shown to reach sub-ppt level noise equivalent concentration with a $10 \mathrm{~cm}$ long sample cell. ${ }^{18}$ Currently, the typical low-volume PAS instruments have sample volumes in the ten to few millimeters range ${ }^{18-20}$ with some realizations in the sub-milliliters range, ${ }^{21,22}$ or at least potential for these small volumes. ${ }^{23,24}$ However, a combination of high detection sensitivity and volume of some tens of microliter, as desirable for coupling with a GC capillary, has to be realized. It should be noted that there have not been many significant attempts to reduce the sample volume of a PAS cell, because there has been no compelling need for such a development.

Other significant advantages of photoacoustics in infrared spectroscopy are that the detector performs equally well independent of the wavelength region and requires no cooling as opposed to the high-sensitivity detectors in FTIR. Due to this, PAS has seen wide use in the broadband analysis of solids and liquids, where it has been used to replace the ordinary detector in an FTIR. ${ }^{25}$ More recently, PAS has been applied to the sensitive high-resolution analysis of trace gases with broad and narrow spectral features using modern broadband laser light sources, ${ }^{26-28}$ as well as various broadly tunable lasers. ${ }^{29-33}$

In this article, we demonstrate a new method to analyze complex mixtures of large molecules by combining state-of-theart LAS with chromatographic separation. We couple a CEPAS detector with a GC for compound separation and with a widely tunable external-cavity quantum cascade laser for compound identification in the optical fingerprint region. We call this hyphenation GC-PAS. We present a proof-of-principle demonstration of the concept and analyze a mixture of eight VOCs ranging from one to eight carbon atoms per compound. 


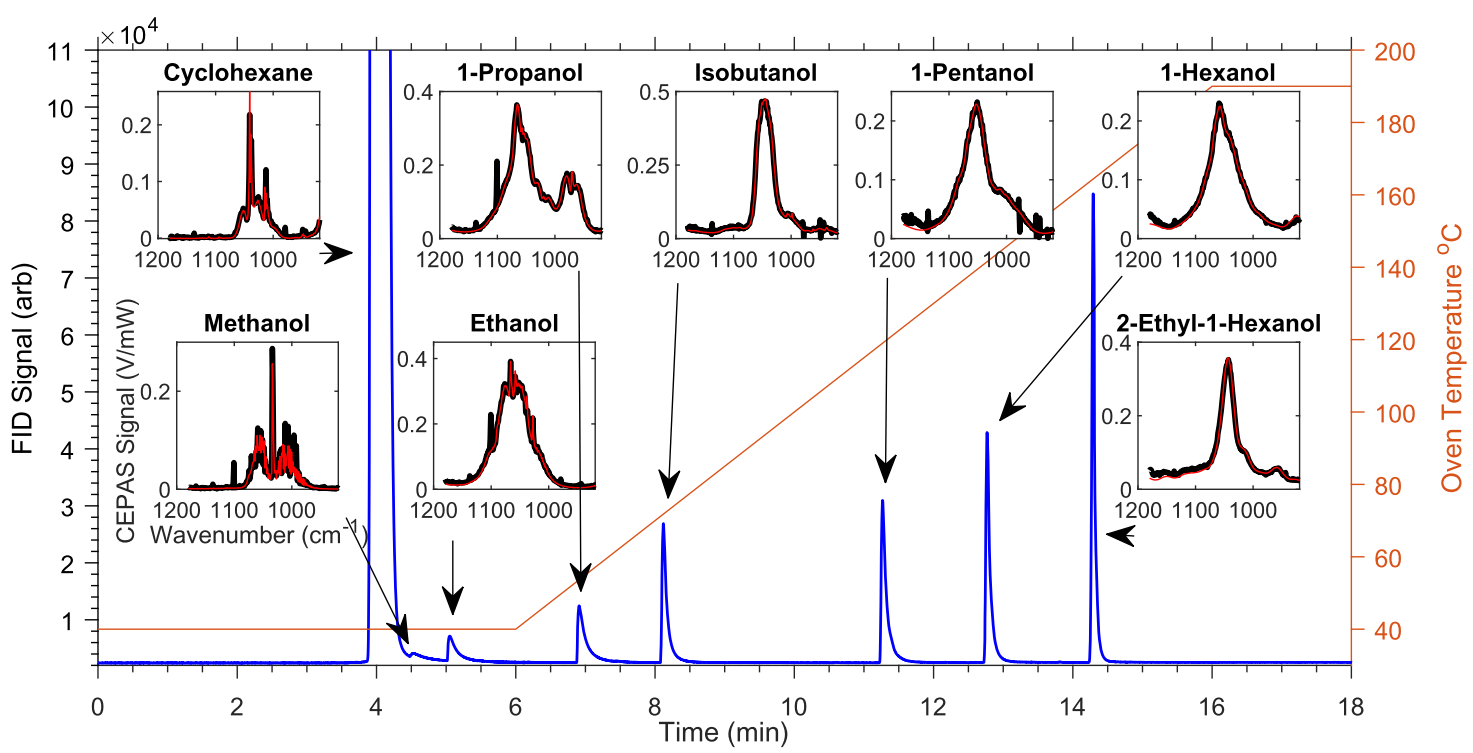

Figure 2. Sample chromatogram (left vertical axis) and the corresponding oven program (right vertical axis). The insets show the corresponding optical-power-normalized CEPAS detector signal (spectra) for each peak. The black traces are the measured data and the red traces are fits to data from the PNNL database. The cyclohexane spectrum is from a different sample solution with a concentration of about 50 times higher than the concentrations of the alcohols in the test solution.

Already at this early stage, the quasi-online detection performance is approximated to be an order of magnitude better than that of an FTIR lightpipe detector. An optimally designed and engineered CEPAS detector could improve the detection limits considerably.

\section{EXPERIMENTAL SECTION}

A schematic picture of the GC-PAS system is given in Figure 1. An HP 5890 Series II gas chromatograph with a polar SigmaAldrich SLB-IL60 column $(30 \mathrm{~m} \times 0.25 \mathrm{~mm} \times 0.2 \mu \mathrm{m}$ film thickness, ionic liquid stationary phase) with $1 \mathrm{~m}$ long deactivated retention gap (inner diameter $0.53 \mathrm{~mm}$, Agilent) was used for the chromatographic separation. The column flow rate was $0.8 \mathrm{~mL} / \mathrm{min}$ (with $\mathrm{N}_{2}$ carrier gas), and the split ratio was $1: 10$. The temperature of the injector was $250{ }^{\circ} \mathrm{C}$, and the GC oven had a variable temperature program from 40 to 190 ${ }^{\circ} \mathrm{C}$, as shown in Figure 2. The sample was manually injected using the hot needle rapid injection technique with a typical injection volume of $0.5 \mu \mathrm{L}$. The chromatograph had a 10-port 2-position valve to direct the effluent of the column to a flame ionization detector (FID) at $300{ }^{\circ} \mathrm{C}$ either directly or through a sample loop, which was a deactivated silica capillary with a 2 $\mathrm{m}$ length and $0.53 \mathrm{~mm}$ inner diameter. Passing the column output through the sample loop allowed a portion of the column effluent to be extracted for detection at the CEPAS detector in a quasi-online manner, as the detector does not operate with a flow through. The maximum size of the effluent portion was $30 \mathrm{~s}$ or $0.44 \mathrm{~mL}$ in volume, which is adequate when compared to the typical 5-6 s FWHH of the eluted peaks. The compact CEPAS detector is a modified version of the Gasera PA201 photoacoustic analyzer. Together with the gas-exchange system and the laser, it could be packed in a portable shoe-box size enclosure requiring no consumables.

The molecular mixture injected through the sample loop, traveled to the CEPAS detector via a $1 \mathrm{~m}$ long transfer line (deactivated silica capillary, $0.32 \mathrm{~mm} \mathrm{ID),} \mathrm{and} \mathrm{heated} \mathrm{to} 150$ ${ }^{\circ} \mathrm{C}$. The carrier, or make-up gas, at this stage, was dry air (about $9000 \mathrm{ppm}$ absolute humidity) at the $8.6 \mathrm{~mL} / \mathrm{min}$ flow rate. At the CEPAS detector, the sample was first collected to an evacuated buffer cell ( $1.28 \mathrm{~mL}, 6 \mathrm{mbar}$ initial pressure $)$ and filled with the make-up gas to 1800 mbar pressure. The purpose of the buffer cell was to homogenize the sample before transferring it to the CEPAS measurement cell (total volume of $11 \mathrm{~mL}, 6 \mathrm{mbar}$ initial pressure). More details about the buffer cell and the gas exchange are in the Supporting Information in part ESI-2. The sample was transferred to the CEPAS measurement cell by opening the connecting valve and letting the pressures stabilize. The end pressure at the equilibrium was about 200 mbar. Before each measurement, the cells were flushed clean with dry air or $\mathrm{N}_{2}$. The flush flow rate was 375 $\mathrm{mL} / \mathrm{min}$. In our experiments, the sample was rejected after the detection. Since PAS is a non-destructive detection method, it would be possible to pass the sample to another detector, although at diluted concentration because of the make-up gas.

Measurement of the extracted gas sample in the CEPAS cell involved directing a laser beam through the cell. The absorption path length was $95 \mathrm{~mm}$ and the diameter of the aperture was $4 \mathrm{~mm}$. To record an infrared spectrum, the continuous wave laser (HedgeHog HHG-41095-UT EC-QCL by Daylight Solutions) wavenumber was scanned in a stepwise manner from 1200 to $900 \mathrm{~cm}^{-1}$ at a $1 \mathrm{~cm}^{-1}$ step size and $4 \mathrm{~Hz}$ step rate. A thermoelectric cooling system kept the laser temperature at $19{ }^{\circ} \mathrm{C}$, while the laser current was held at 1.65 A. The maximum laser power was $130 \mathrm{~mW}$. Before passing the cell, the optical power of the laser was modulated on/off by an optical chopper wheel at the $85 \mathrm{~Hz}$ rate for the generation of the photoacoustic signal. Pulsed light in the PA cell creates periodic heating through absorption, which converts to a periodic pressure change, an acoustic wave in a closed space. The PA signal was detected by a sensitive microphone, a silicon cantilever-based interferometer, ${ }^{34}$ and recorded using a lock-in detection at the modulation frequency. A spectral scan took $75 \mathrm{~s}$, while loading the sample gas into the cell took $35 \mathrm{~s}$. Flushing the cell clean required a few minutes, depending on the boiling points of the analytes. The optical power of the laser was continuously monitored after the cell for normal- 
ization purposes as the PA signal is directly proportional to optical power. An optical power measurement after the cell accurately represents the intracell power and is also the most convenient way to monitor the power. The power was recorded on each measurement, with no noticeable fluctuations over the course of the experiment. The CEPAS cell windows were antireflection-coated $\mathrm{ZnSe}$ windows (Thorlabs WG70530-G) to minimize reflections of the laser beam and, therefore, the background signal resulting from power modulation of the light source.

\section{RESULTS AND DISCUSSION}

To test our GC-PAS system, we prepared a solution of several alcohols in cyclohexane at about $0.26 \% \mathrm{~mol} / \mathrm{mol}$ concentration each. Alcohols exhibit characteristic infrared signatures of the $\mathrm{C}-\mathrm{OH}$ stretching vibration in the middle of the spectral scan range, which makes them easy to detect but impossible to resolve without chromatographic separation.

Figure 2 shows a chromatogram of the test solution. The methanol peak slightly overlaps with the tail of the solvent peak. Otherwise, the peaks are well-resolved, permitting unambiguous sampling for the CEPAS detector. The tailing of the analytes, which is stronger for the smaller more polar alcohols, is attributed to the slight incompatibility with the solvent. The insets in Figure 2 show the corresponding CEPAS detector signals (spectra) for each peak. Black traces are the measured data, which have a background spectrum (no sample) subtracted and divided by a signal from the optical power meter. Red traces are classical linear least squares (CLS) fits using the Pacific Northwest National Laboratory (PNNL) data of the compounds. ${ }^{35}$ The data were measured with dry air as a make-up gas. The fits show that the measured spectra match well with the PNNL database results and that it is possible to use the existing spectral libraries directly to identify compounds. For routine measurements, however, it is recommended to collect a separate spectral library, which more closely represents the fine details of the detection system. These details, such as adsorption and poor mixing of the gases, are discussed in the Supporting Information in part ESI-2.

For the most accurate CLS analysis, a model library was built for the compounds of interest by recording each spectrum at a high signal-to-noise ratio (SNR) from a prepared internal calibration standard that was run and sampled through the GC. To further improve the CLS performance, we have excluded $25 \%$ of the spectral data points that were noisy because of sharp spectral features caused mainly by $\mathrm{H}_{2} \mathrm{O}$ and $\mathrm{CO}_{2}$ in the sample. The excluded experimental data points are included in the insets of Figure 2 with the purpose of presenting the raw data.

The present proof-of-concept implementation of the CEPAS detector for GC has a slow gas-exchange cycle, which does not allow real-time sampling of a chromatogram. In the presented results, we have extracted one sample from each GC run. In Figure 2, each spectrum has been recorded on a different GC run, by extracting the whole eluted peak to the CEPAS detector. Although extracting several samples in 2 min intervals is technically possible even with the current setup, it does not leave enough time for cleaning thoroughly the detector gas cell from a previous sample before a new one arrives. This would introduce spectral interference and reduce selectivity, especially at low signal levels.

In general, photoacoustic detectors can be operated in the flow-through mode for rapid gas analysis. A prerequisite for a good instrument performance is, however, that the acoustic cell is properly designed to suppress the flow noise. ${ }^{36}$ Some photoacoustic methods, such as QEPAS, are also relatively insensitive to environmental noise and thus better suited for flow through. ${ }^{37}$ The employed CEPAS detector does not follow these design approaches because the best detection sensitivity is achieved with a closed cell; hence, it is often the best choice for trace-level analysis.

Whereas transmission spectroscopy can provide pseudoabsolute quantification in $\mathrm{GC}^{38}$ photoacoustic spectroscopy requires a traditional calibration of the detector response. This is carried out in relation to the injected sample. We performed the calibration using the same measurements that we employed for recording the spectral library, together with a background measurement. The spectrum of each compound was measured once, and a calibration coefficient was calculated for a spectrum with the background measurement subtracted. In addition, we checked the linearity of the CEPAS detector for some of the compounds by diluting the alcohol mixture to $1: 5$, $1: 10$, and 1:20 ratios and performing the measurements again. To correct for inaccuracy introduced by the manual injection technique, we corrected the sample amounts by the recorded FID signal, which is known to be linear. The results are presented in Supporting Information Figure S2. The analyzed concentration range of more than an order of magnitude reflects the current application range of the detector. The largest sample amounts are about 5 times smaller than the saturation limit of the detector, while the smallest values are close to the limits of quantification. The intrinsic dynamic range of the detector is about 5 orders of magnitude, which is commonly achieved in the wavelength-modulated trace gas detection of small molecules with this detector. In this work, the intrinsic detection limit was not yet reached because the nonoptimized design caused excess experimental noise.

Spectroscopic detection expands the chromatographic measurement to a two-dimensional separation. Spectroscopy can be used for two purposes in GC-PAS: to identify and quantify the presence of compounds in an eluted peak based on their characteristic spectra. In most cases, this is possible even in the case of simultaneously eluted compounds. Chemometrics offer tools for such purpose, one of which is CLS, allowing multicomponent analysis based on a spectral library. ${ }^{39}$ In Figures 3 and 4, we give examples of the usefulness of CLS in identifying and quantifying multiple compounds simultaneously.

In Figure 3, we show a 1-pentanol measurement (15.8 ng on-column amount, a $1.9 \mathrm{ppm}$ volume mixing ratio in the CEPAS cell) in which another gas species of about the same absorption strength unintentionally interferes with the 1pentanol spectrum. The interferents appear when the injector purge is switched off, suggesting septum bleed as the cause. The chosen column has a low-bleed ionic liquid stationary phase (not a polysiloxane phase). Septum and column bleeds are common problems in gas chromatography, affecting the background stability and detection limits. ${ }^{40}$ In the case of a spectroscopic detector, a high enough bleed could impede the spectral interpretation. The bleed products are often cyclic siloxanes. A spectral library search reveals that the two major septum bleed products are, in this case, $\mathrm{D}_{3}$ and $\mathrm{D}_{5}$ (see Figure 3 caption). The identification was performed on a measurement with only the interferents present. Figure 3 demonstrates how adding these two components to the CLS analysis results in a correct spectral fit for the main analyte 1-pentanol, 


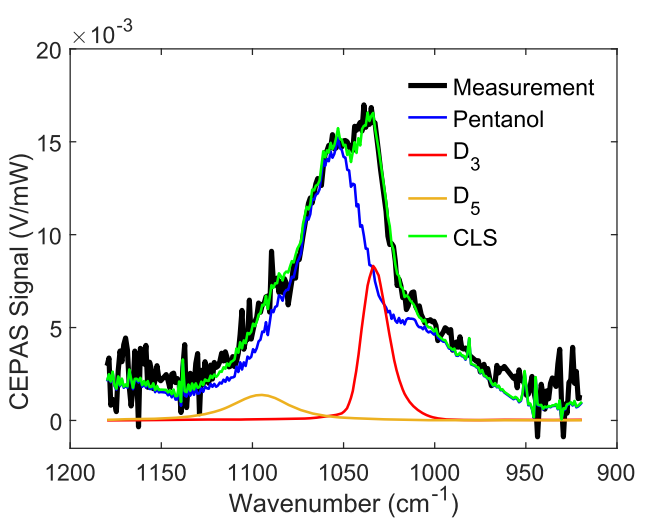

Figure 3. 1-Pentanol with interfering gas species. $\mathrm{D}_{3}$ : hexamethylcyclotrisiloxane, $\mathrm{D}_{5}$ : decamethylcyclopentasiloxane, CLS: classical linear least squares fit.

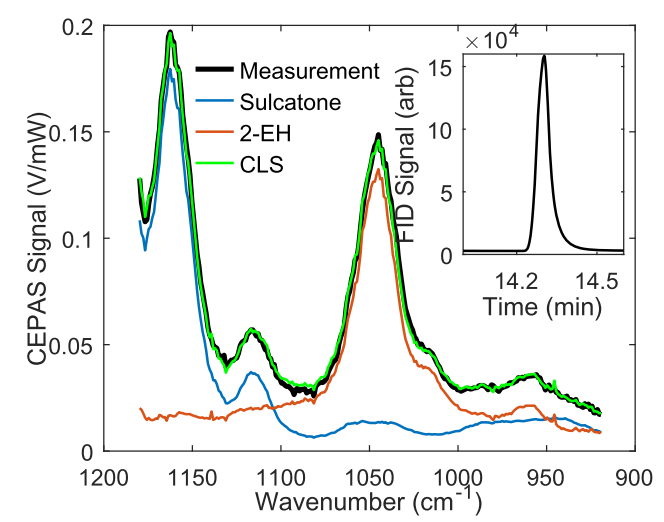

Figure 4. Overlapping GC peaks (inset) spectrally resolved with the CEPAS detector. 2-EH: 2-ethyl-1-hexanol, CLS: classical linear least squares fit.

avoiding erroneous detection because of the bleed products. It is worthwhile to note that the vibrational $\mathrm{Si}-\mathrm{O}-\mathrm{Si}$ band in the $1130-1000 \mathrm{~cm}^{-1}$ region is very strong, about $30-50$ times stronger than the absorption bands of the alcohol in the same wavenumber region, ${ }^{35,41}$ which directly translates to improved detection limits for siloxanes.

In Figure 4, we show an example of a case where the eluted peaks of two species, sulcatone (6-methyl-5-hepten-2-one) and 2-ethyl-1-hexanol, completely overlap using an FID, as seen in the inset in Figure 4. Spectrally, the two are well-resolved using CEPAS and CLS, demonstrating the benefit from a twodimensional separation. Figure S3 in the Supporting Information shows separate measurements of sulcatone and 2-EH with the FID.

Photoacoustic spectroscopy is intrinsically a background-free method. If there is no absorbing gas in the PA cell, there is no PA signal. However, windows enclosing the cell are never perfectly transmissive and absorption on the window material can create a PA signal. The windows also scatter light onto the cell walls, which again absorb and create a PA signal. Normally, in laser photoacoustic spectroscopy, such broadband absorption is not a problem since one could use wavelength modulation (WM) techniques to probe the narrow spectral features of small molecules of interest. ${ }^{42}$ In the case of large molecules, the lack of such narrow features prevents the use of WM techniques. Instead, photoacoustic modulation needs to be induced by pulsing the optical power of the laser on/off using an optical chopper wheel, which results in a background signal. In our experiment, the background signal level is about $3 \%$ of the full dynamic range of the CEPAS detector. Most of the background signal is estimated to originate from absorption in the window material.

The background noise often limits the achievable limit of detection (LOD), which is also the case with the CEPAS detector. In our experiment, we have determined the LOD for test compounds by performing repeated background measurements, subtracting the average background from the measurements, and analyzing the sensitivity of each CLS model to the residual background fluctuations. We define LODs as 3 times the standard deviation $(3 \sigma)$ of the CLS analysis results in each case. These LODs are summarized in the first two columns of Table 1, under the title "Background $\mathrm{N}_{2}$ ". We used $\mathrm{N}_{2}$ as

Table 1. Estimated Limits of detection. The Values under the Title "Background $\mathrm{N}_{2}$ " Describe the Actual Experimental Performance, While the "CEPAS Limited" Values Indicate the Intrinsic Noise Limit of the Detector. The Units are Expressed as On-Column Masses, and as Photoacoustic Cell Volume Mixing Ratios

\begin{tabular}{lrrrrr} 
& \multicolumn{2}{c}{ background $\mathrm{N}_{2}$} & & \multicolumn{2}{c}{ CEPAS limited } \\
\cline { 2 - 3 } \cline { 5 - 6 } compound & \multicolumn{1}{c}{$\mathrm{pg}$} & $\mathrm{ppb}$ & & $\mathrm{pg}$ & $\mathrm{ppb}$ \\
cyclohexane & 7540 & 902 & & 458 & 55 \\
methanol & 181 & 57 & & 12 & 4 \\
octane & 19000 & 1670 & & 792 & 70 \\
ethanol & 292 & 64 & & 16 & 3 \\
acetone & 6170 & 1070 & & 261 & 45 \\
1-propanol & 438 & 73 & & 19 & 3 \\
isobutanol & 325 & 44 & & 18 & 2 \\
1-pentanol & 956 & 109 & & 46 & 5 \\
1-hexanol & 1110 & 109 & & 54 & 5 \\
2-EH & 2090 & 161 & & 92 & 7 \\
\hline
\end{tabular}

make-up and flush gas because it gives a more stable background than the unpurified pressurized air feed. In addition, we studied what the achievable LOD could be by eliminating some of the major sources of uncertainty in the future. As an example, the CEPAS limited LODs in Table 1 are determined using background noise measurements with the laser turned off, indicating the achievable performance if the laser noise and sampling fluctuations are eliminated. This limit can typically be reached in WM spectroscopy of small molecules when the gas exchange and laser power noise do not significantly affect the measurement. The large difference in LODs of, for example, octane and propanol is mainly due to the difference in the respective absorption cross sections in the wavelength region of the laser. The stronger absorbance of propanol results in a better LOD compared to that of octane. The full table of the findings together with additional discussion is presented in Supporting Information ESI-1.

As can be seen from Table 1, currently the detection performance of the GC-PAS instrument is limited by the fluctuations and noise coming from the sampling and the laser, not from the intrinsic noise of the detector. Beyond that, the limiting factor is still the relatively large volume of the CEPAS cell $(11 \mathrm{~mL})$. As the CEPAS detector operates quasi-online, the smallest useful size of the cell would be equal to the volume of a GC peak, as opposed to the full width at half-height volume for online detectors. For the experimental setup presented here, the full peak volume is about $0.2 \mathrm{~mL}$. 
Therefore, we currently lose a factor in the order of 55 in sensitivity using a make-up gas to match the size of the sample and the cell volume. Reducing the cell volume would also make the measurement at higher pressure (column pressure) feasible. This would allow faster gas-exchange cycles and greater absorption strength. An optimal cell volume could be as low as few hundred microliters, which is close to a typical volume for an optical GC detector. Another point to consider for future improvements is that our current CEPAS detector can operate only up to $50{ }^{\circ} \mathrm{C}$, making the handling of highboiling-point compounds difficult as they tend to stick on the walls of the cell. However, an earlier study has shown that the CEPAS detector can operate at least up to $180{ }^{\circ} \mathrm{C}$ with suitable engineering. ${ }^{43}$ Overall, an optimally designed CEPAS detector could improve the detection limits of GC-PAS as much as 2-3 orders of magnitude, resulting in low-pg to sub-pg LODs and thus surpassing FID performance also in terms of detection limits, not only in selectivity.

\section{CONCLUSIONS}

We have demonstrated a novel GC-PAS hyphenated technique, which offers considerable advantages for analyzing complex mixtures of large molecules, especially in applications that require portability. As an infrared spectroscopic technique, GC-PAS provides extensive molecular identification capability. FTIR lightpipe detectors have LODs commonly in the order of ng or tens of ng. ${ }^{1,2}$ Our proof-of-principle setup reaches LODs of 200-2000 pg for the alcohols, which had an optimal absorption spectrum with respect to the wavelength range of our broadly tunable laser. Therefore, already in the present form, GC-PAS offers a detection sensitivity that is on average an order of magnitude better than GC-FTIR. Compared to FID, the spectroscopy adds another dimension to the analysis. This improves the selectivity without requiring any consumables to operate, as purified air can be used as a carrier gas and the sample injection can be automated by the use of an injector valve and a sample loop. Compared to MS, PAS provides a more straightforward interpretation of the results, is easily packed to a small size, and is thus suitable for field instruments for nonexperts. An example of an application that would benefit from our approach is indoor air quality studies, in which 2-ethyl-1-hexanol is a widely recognized indicator of an air quality problem. ${ }^{44}$ The GC-PAS could allow relatively fast on-site measurements of 2-ethyl-1-hexanol, which would be a significant advancement to the prolonged laboratory analysis currently in use.

In our proof-of-principle demonstration of GC-PAS, we employed a state-of-the-art cantilever-enhanced photoacoustic spectroscopic detector together with a widely tunable singlefrequency EC-QCL laser to analyze a mixture of alcohols in a quasi-online manner. The sample matrix was complicated enough that chromatographic separation or infrared analysis alone could not have satisfactorily distinguished all of the components of the mixture. Even though the PA detector module was nonoptimized for operation in conjunction with the GC, we were able to reach improved detection limits compared to what is possible with FTIR lightpipe detectors. The remaining technical challenges are connected with the temperature range of the CEPAS cell, slow gas exchange, and the static measurement, which is a problem for adsorptive molecules. Our new approach fully utilizes the capabilities of infrared spectroscopy in molecular identification and multicomponent analysis in the case of unresolved GC peaks, as well as in the presence of interference caused by a column or septum bleed.

Photoacoustic spectroscopy is not the only potential laserbased infrared detection method for GC. As an alternative approach, hollow optical waveguides, ${ }^{9,10,39}$ including hollowcore photonic crystal fibers, ${ }^{45}$ offer an efficient way of minimizing the sample volume. Additionally, miniaturization of optical cavities could make cavity-enhanced absorption spectroscopic techniques a tempting detection method for GC. Cavity-enhanced absorption spectroscopy is also suitable for broadband infrared spectroscopy with modern laser light sources, such as optical frequency combs and supercontinuums. $^{46,47}$

It is very difficult to surpass the performance of GC-MS in a standard laboratory environment with the possible exception of some niche applications. However, already in the present form, the GC-PAS hyphenated technique introduced in this article could be developed into an efficient field instrument for specific applications, as the size, cost, and requirement of no consumables are greatly in favor of GC-PAS.

\section{ASSOCIATED CONTENT}

\section{Supporting Information}

The Supporting Information is available free of charge at https://pubs.acs.org/doi/10.1021/acs.analchem.0c02887.

Additional data and explanations are available in the Supporting information: limits of detection analysis, gasexchange details, linearity test, and chromatograms of co-eluted peaks (PDF)

\section{AUTHOR INFORMATION}

\section{Corresponding Author}

Markus Metsälä - Department of Chemistry, University of Helsinki, FI-00014 Helsinki, Finland; o orcid.org/00000002-0716-7132; Email: markus.metsala@helsinki.fi

\section{Authors}

Teemu Tomberg - Department of Chemistry, University of Helsinki, FI-00014 Helsinki, Finland; (1) orcid.org/00000003-3448-2880

Niko Vuorio - Department of Chemistry, University of Helsinki, FI-00014 Helsinki, Finland

Tuomas Hieta - Gasera Ltd., FI-20520 Turku, Finland

Matti Jussila - Department of Chemistry, University of Helsinki, FI-00014 Helsinki, Finland

Kari Hartonen - Department of Chemistry, University of Helsinki, FI-00014 Helsinki, Finland; (o orcid.org/00000003-4821-2355

Markku Vainio - Department of Chemistry, University of Helsinki, FI-00014 Helsinki, Finland; Photonics Laboratory, Physics Unit, Tampere University, FI-33014 Tampere, Finland

Tommi Mikkonen - Photonics Laboratory, Physics Unit, Tampere University, FI-33014 Tampere, Finland

Juha Toivonen - Photonics Laboratory, Physics Unit, Tampere University, FI-33014 Tampere, Finland

Marja-Liisa Riekkola - Department of Chemistry, University of Helsinki, FI-00014 Helsinki, Finland; 으이.org/00000001-7519-9892

Lauri Halonen - Department of Chemistry, University of Helsinki, FI-00014 Helsinki, Finland; ㅇorcid.org/00000003-4637-2689

Complete contact information is available at: 
https://pubs.acs.org/10.1021/acs.analchem.0c02887

\section{Author Contributions}

The manuscript was written through contributions of all authors. All authors have given approval to the final version of the manuscript.

Notes

The authors declare no competing financial interest.

\section{ACKNOWLEDGMENTS}

We acknowledge the following funding sources: CHEMS doctoral program of the University of Helsinki, Doctoral School of Tampere University, Academy of Finland Flagship Programme, Photonics Research and Innovation (PREIN) (320165), and Academy of Finland (Grants 314363 and 294752).

\section{REFERENCES}

(1) Zavahir, J. S.; Nolvachai, Y.; Marriott, P. J. TrAC, Trends Anal. Chem. 2018, 99, 47-65.

(2) Griffiths, P. R.; Heaps, D. A.; Brejna, P. R. Appl. Spectrosc. 2008, $62,259 \mathrm{~A}-70 \mathrm{~A}$.

(3) Schug, K. A.; Sawicki, I.; Carlton, D. D., Jr.; Fan, H.; McNair, H. M.; Nimmo, J. P.; Kroll, P.; Smuts, J.; Walsh, P.; Harrison, D. Anal. Chem. 2014, 86, 8329-8335.

(4) Lelevic, A.; Souchon, V.; Moreaud, M.; Lorentz, C.; Geantet, C. J. Sep. Sci. 2020, 43, 150-173.

(5) Schliesser, A.; Picqué, N.; Hänsch, T. W. Nat. Photonics 2012, 6, $440-449$.

(6) Hugi, A.; Maulini, R.; Faist, J. Semicond. Sci. Technol. 2010, 25, No. 083001 .

(7) Muraviev, A. V.; Smolski, V. O.; Loparo, Z. E.; Vodopyanov, K. L. Nat. Photonics 2018, 12, 209-214.

(8) Hugi, A.; Terazzi, R.; Bonetti, Y.; Wittmann, A.; Fischer, M.; Beck, M.; Faist, J.; Gini, E. Appl. Phys. Lett. 2009, 95, 1103.

(9) Wu, S.; Deev, A.; Haught, M.; Tang, Y. J. Chromatogr. A 2008, 1188, 327-330.

(10) Mengali, S.; Liberatore, N.; Luciani, D.; Viola, R.; Cardinali, G. C.; Elmi, I.; Poggi, A.; Zampolli, S.; Biavardi, E.; Dalcanale, E.; Bonadio, F.; Delemont, O.; Esseiva, P.; Romolo, F. Rapid screening and identification of illicit drugs by IR absorption spectroscopy and gas chromatography, SPIE OPTO, Razeghi, M., Ed.; SPIE, 2013; pp 489498.

(11) Zare, R. N.; Kuramoto, D. S.; Haase, C.; Tan, S. M.; Crosson, E. R.; Saad, N. M. R. Proc. Natl. Acad. Sci. U.S.A. 2009, 106, 1092810932.

(12) Zharov, V.; Montanari, S.; Letokhov, V. Laser Chemistry 1983, $1,163-176$.

(13) Kreuzer, L. B. Anal. Chem. 1978, 50, 597A-606A.

(14) Xiong, L.; Bai, W.; Chen, F.; Zhao, X.; Yu, F.; Diebold, G. J. Proc. Natl. Acad. Sci. U.S.A. 2017, 114, 7246-7249.

(15) Dumitras, D. C.; Dutu, D. C.; Matei, C.; Magureanu, A. M.; Petrus, M.; Popa, C. J. Optoelectron. Adv. Mater. 2007, 9, 3655-3701.

(16) Patimisco, P.; Scamarcio, G.; Tittel, F. K.; Spagnolo, V. Sensors 2014, 14, 6165-6206.

(17) Spagnolo, V.; Patimisco, P.; Borri, S.; Scamarcio, G.; Bernacki,

B. E.; Kriesel, J. Opt. Lett. 2012, 37, 4461-4463.

(18) Tomberg, T.; Vainio, M.; Hieta, T.; Halonen, L. Sci. Rep. 2018 , 8, No. 1848.

(19) Yang, X.; Xiao, Y.; Ma, Y.; He, Y.; Tittel, F. K. Sensors 2017, 17, No. 1750.

(20) Rouxel, J.; Coutard, J. G.; Gidon, S.; Lartigue, O.; Nicoletti, S.; Parvitte, B.; Vallon, R.; Zéninari, V.; Glière, A. Proc. Eng. 2015, 120, 396-399.

(21) Gorelik, A. V.; Ulasevich, A. L.; Nikonovich, F. N.; Zakharich, M. P.; Firago, V. A.; Kazak, N. S.; Starovoitov, V. S. Appl. Phys. B 2010, 100, 283-289.
(22) Gorelik, A. V.; Ulasevich, A. L.; Kuz'muk, A. A.; Starovoitov, V. S. Opt. Spectrosc. 2013, 115, 567-573.

(23) Gruca, G.; Heeck, K.; Rector, J.; Iannuzzi, D. Opt. Lett. 2013, $38,1672-1674$.

(24) Zhou, S.; Slaman, M.; Iannuzzi, D. Opt. Express 2017, 25, $17541-17548$.

(25) Michaelian, K. H. Photoacoustic IR Spectroscopy: Instrumentation, Applications and Data Analysis; John Wiley \& Sons, 2010.

(26) Karhu, J.; Tomberg, T.; Senna Vieira, F.; Genoud, G.; Hänninen, V.; Vainio, M.; Metsälä, M.; Hieta, T.; Bell, S.; Halonen, L. Opt. Lett. 2019, 44, 1142-1145.

(27) Mikkonen, T.; Amiot, C.; Aalto, A.; Patokoski, K.; Genty, G.; Toivonen, J. Opt. Lett. 2018, 43, 5094-5097.

(28) Sadiek, I.; Mikkonen, T.; Vainio, M.; Toivonen, J.; Foltynowicz, A. Phys. Chem. Chem. Phys. 2018, 20, 27849-27855.

(29) Larnimaa, S.; Halonen, L.; Karhu, J.; Tomberg, T.; Metsälä, M.; Genoud, G.; Hieta, T.; Bell, S.; Vainio, M. Chem. Phys. Lett. 2020, 750 , No. 137488

(30) Mammez, D.; Stoeffler, C.; Cousin, J.; Vallon, R.; Mammez, M. H.; Joly, L.; Parvitte, B.; Zéninari, V. Infrared Phys. Technol. 2013, 61, 14-19.

(31) Berer, T.; Brandstetter, M.; Hochreiner, A.; Langer, G.; Märzinger, W.; Burgholzer, P.; Lendl, B. Opt. Lett. 2015, 40, 34763479.

(32) Phillips, M. C.; Myers, T. L.; Wojcik, M. D.; Cannon, B. D. Opt. Lett. 2007, 32, 1177-1179.

(33) Mukherjee, A.; Dunayevskiy, I.; Prasanna, M.; Go, R.; Tsekoun, A.; Wang, X.; Fan, J.; Patel, C. K. N. Appl. Opt. 2008, 47, 1543-1548.

(34) Kuusela, T.; Kauppinen, J. Appl. Spectrosc. Rev. 2007, 42, $443-$ 474 .

(35) Sharpe, S. W.; Johnson, T. J.; Sams, R. L.; Chu, P. M.; Rhoderick, G. C.; Johnson, P. A. Appl. Spectrosc. 2004, 58, 14521461.

(36) Kapitanov, V. A.; Ponomarev, Y. N.; Song, K.; Cha, H. K.; Lee, J. Appl. Phys. B 2001, 73, 745-750.

(37) Kosterev, A. A.; Tittel, F. K. Appl. Opt. 2004, 43, 6213-6217.

(38) Bai, L.; Smuts, J.; Walsh, P.; Qiu, C.; McNair, H. M.; Schug, K. A. Anal. Chim. Acta 2017, 953, 10-22.

(39) Perez-Guaita, D.; Wilk, A.; Kuligowski, J.; Quintás, G.; de la Guardia, M.; Mizaikoff, B. Anal. Bioanal. Chem. 2013, 405, 82238232.

(40) Jennings, W.; Mittlefehldt, E.; Stremple, P. Analytical Gas Chromatography; Academic Press, 1997.

(41) Bernard, F.; Papanastasiou, D. K.; Papadimitriou, V. C.; Burkholder, J. B. J. Quant. Spectrosc. Radiat. Transfer 2017, 202, 247254.

(42) Schilt, S.; Thévenaz, L. Infrared Phys. Technol. 2006, 48, 154162.

(43) Hirschmann, C. B.; Koivikko, N. S.; Raittila, J.; Tenhunen, J.; Ojala, S.; Rahkamaa-Tolonen, K.; Marbach, R.; Hirschmann, S.; Keiski, R. L. Sensors 2011, 11, 5270-5289.

(44) Nalli, S.; Horn, O. J.; Grochowalski, A. R.; Cooper, D. G.; Nicell, J. A. Environ. Pollut. 2006, 140, 181-185.

(45) Yao, C.; Wang, Q.; Lin, Y.; Jin, W.; Xiao, L.; Gao, S.; Wang, Y.; Wang, P.; Ren, W. Opt. Lett. 2019, 44, 4048-4051.

(46) Foltynowicz, A.; Masłowski, P.; Fleisher, A. J.; Bjork, B. J.; Ye, J. Appl. Phys. B 2013, 110, 163-175.

(47) Amiot, C.; Aalto, A.; Ryczkowski, P.; Toivonen, J.; Genty, G. Appl. Phys. Lett. 2017, 111, No. 061103. 